\title{
Conocimientos biológicos de la homosexualidad y transexualidad en estudiantes de Educación Física de Chile \\ Biological knowledge of homosexuality and transsexuality in Physical Education students in Chile
}

\author{
Fernando Maureira Cid \\ Universidad Metropolitana de Ciencias de la Educación (Chile)
}

\begin{abstract}
Resumen. Introducción: la homosexualidad y la transexualidad están mediadas por mecanismos biológicos de tipo genético, accionar temprano de hormonas y por mecanismos ambientales, lo que repercute en cambios estructurales y funcionales de diversas regiones cerebrales. El objetivo de la presente investigación es describir los conocimientos biológicos de la homosexualidad y transexualidad de estudiantes de Educación Física. Método: se construyó y valido un cuestionario diseñado ad hoc a 305 estudiantes de Pedagogía en Educación Física de tres universidades de Chile. Resultados: el cuestionario quedó constituido por 18 ítems, además presenta seis factores que explican el 58.378\% de la varianza y un alfa de Cronbach de .763. El ítem que presentó mayor cantidad de respuestas acertadas fue Existe un "gen gay» que determina la homosexualidad y el lesbianismo y el que presento menor cantidad de aciertos fue Cuando una mujer es lesbiana existen más probabilidad que su hermana también sea lesbiana. Conclusión: los análisis demuestran que el instrumento es válido y confiable para evaluar el conocimiento biológico de la homosexualidad y transexualidad en estudiantes de Pedagogía en Educación Física. En 14 ítems más del 50\% de la muestra contesto no sé y el 78.6\% de la muestra obtuvo sólo entre una y seis respuestas correctas.
\end{abstract}

Palabras claves: biología, cerebro, homosexualidad, transexualidad, educación física.

\begin{abstract}
Introduction: homosexuality and transsexuality are mediated by biological mechanisms of genetic type, early action of hormones and by environmental mechanisms, affecting structural and functional changes in various brain regions. The aim of this research is describe the biological knowledge of homosexuality and transsexuality for students of Physical Education. Method: an ad hoc questionnaire was built and applied to 305 students of Pedagogy in Physical Education from three universities in Chile. Results: the questionnaire was constituted by 18 items, in addition it presents six factors that explain the $58.378 \%$ of variance and a Cronbach alpha of .763. The item that presented the most successful answers was There is a "gay gene» that determines homosexuality and lesbianism and the one that presents the least number of successful was When a woman is a lesbian there is more chance that her sister is also a lesbian. Conclusion: Analyses show that the instrument is valid and reliable for assessing the biological knowledge of homosexuality and transsexuality in students of Physical Education Pedagogy. In 14 items more than $50 \%$ of the sample answered $I$ do not know and $78.6 \%$ of the sample obtained only between one and six correct answers.
\end{abstract}

Keywords: biology, brain, homosexuality, transsexuality, physical education.

\section{Introducción}

La orientación sexual corresponde a una dirección de sentimientos y conductas sexuales hacia alguien de sexo opuesto (heterosexual), hacia el mismo sexo (homosexual), ambos sexos (bisexual) o ninguno (asexual). Esto involucra la atracción afectiva, romántica y sexual (American Psychological Association, 2011). La orientación sexual esta mediada por tres mecanismos: acciones directas de genes específicos del sexo, acciones tempranas de esteroides sexuales y mecanismo epigenéticos (Balthazart, 2020). En las últimas décadas se ha profundizado en los estudios que buscan las bases biológicas de

Fecha recepción: 04-04-21. Fecha de aceptación: 30-05-21

Fernando Maureira

maureirafernando@yahoo.es la orientación sexual, dando luz sobre algunos aspectos hormonales y cerebrales que parecen involucrados en estos procesos.

Se han observado diversas preferencias sexuales en varios modelos animales, resaltando los carneros que presentan entre 6-10\% de machos que copulan con otros machos (Annicchiarico, 2009), los macacos japoneses con altos índices de actividad sexual con individuos de ambos sexos (Vasey, et al., 2006), las ratas donde algunos machos copulan con otro machos (Coria-Ávila, 2012) y los monos bonobos, donde el 60\% de las hembras presentan actividad sexual con otras hembras (Clay y de Waal, 2015; Clay y Zuberbühler, 2011). También se han observado conductas sexuales hacia individuos del mismo sexo en delfines, hienas, leones, perros, patos, pingüinos, gaviotas, palomas, lagartijas, moscas y libélulas, 
con descripciones en más de 450 especies (Bagemihl, 1999).

Las investigaciones sobre las orientaciones sexuales en humanos han llevado al estudio de dimorfismos sexuales en cerebros de heterosexuales y homosexuales, siendo el trabajo de Swaab y Hofman (1990) el primero de ellos, donde se describe que el núcleo supraquiasmático de hipotálamo en hombres homosexuales es casi del doble de tamaño que en hombres heterosexuales. LeVay (1991) reportó que el núcleo intersticial del hipotálamo anterior 3 (NIHA-3), que se relaciona con las conductas sexuales, es mayor en hombres heterosexuales que en hombres homosexuales, presentando en los segundos un tamaño similar a las mujeres. Pese a que esto fue cuestionado argumentando que la muestra de homosexuales padecía VIH, estudios posteriores en hombres homosexuales sanos entregaron resultados similares (Hines, 2011).

Otra región dimórficas es la comisura anterior, una parte del cuerpo calloso que une anatómica y funcionalmente ambos hemisferios cerebrales, la cual presenta mayor tamaño en homosexuales (Kreukels \& Guillamon, 2016). Un estudio de Manzouri y Savic (2018) concluye que la homosexualidad parece estar asociada a una menor diferenciación de la sustancia blanca y menor conectividad funcional de algunas regiones cerebrales. También recalcan que los cerebros de hombres heterosexuales y homosexuales presentan mayor diferencia entre sí, que los cerebros de mujeres heterosexuales y lesbianas.

Desde el punto de vista funcional, se han observado variaciones en los sistemas moduladores difusos de dopamina y serotonina según orientaciones sexuales (Kinnunen et al., 2004). El hipotálamo, que incluye el área preóptica medial (APOM), se relaciona con la cópula y su activación depende de la orientación sexual, con una actividad similar en hombres homosexuales y mujeres heterosexuales (Savic et al., 2005). El núcleo estriado ventral, que se relaciona con el deseo sexual, presenta una mayor actividad, frente a estímulos eróticos femeninos, en mujeres lesbianas en comparación con mujeres heterosexuales y bisexuales (Safron et al., 2018).

Las diferencias neuroanatómicas observadas en personas con diferentes orientaciones sexuales se atribuyen a efectos hormonales en el período gestacional (Savic et al., 2010), por ejemplo, la masculinización del cerebro durante la gestación se produce por la acción de la testosterona (Morris et al., 2004). También se ha observado que la hiperplasia suprarrenal congénita produce modificaciones en la biosíntesis de esteroides sexuales en el embrión, con una exposición a elevados niveles de testosterona, lo cual no produce modificaciones en la orientación sexual de hombres, pero entre el 15\% y $30 \%$ de las mujeres presenta mayor excitación, fantasías y comportamientos lésbicos (Wang et al., 2019).

Desde el punto de vista genético, se ha observado que los hermanos de homosexuales también lo son en un $25 \%$, en contraste con hermanos de heterosexuales que solo llegan al 5\%. En el caso de mujeres lesbianas sus hermanas también lo son en un 15\% (Bailey \& Pillard, 1991). Un estudio genético realizado con 493.001 participantes de diversos países revela que no existe un gen gay, sino que la orientación sexual es poligénica, lo que da cuenta de una predisposición hacia la homosexualidad, pero que podría ser modulada por influencias socioculturales (Ganna et al., 2019). Langström et al. (2010) encontraron una influencia genética del 39\% en conductas homosexuales en hombres con hermanos homocigotos y dicigotos homosexuales. En mujeres la influencia fue del 19\%. En la actualidad se siguen estudiando los aspectos biológicos que podrían influir en las orientaciones sexuales, sin dejar de lado los aspectos socioculturales, ya que la heterosexualidad y homosexualidad son fenómenos multifactoriales.

Por otra parte, la identidad de género corresponde a la autopercepción subjetiva que cada individuo tiene sobre su género (Maureira, 2020), es decir, una autoclasificación como hombre o mujer sobre la base de lo que culturalmente se entiende por hombre o mujer (García, 2005). Esta autopercepción puede coincidir con el sexo asignado al nacer (cisgénero) o puede ser diferente (transgénero). En este último caso existen subclasificaciones como la androginia, género no-binario, género fluido, transexualidad, etc. (Landarroitajauregi, 2010; Platero, 2014; Rosalba, 2016, Solvey, 2014).

La identidad de género y la orientación sexual se entrecruzan, con personas cisgénero heterosexuales, homosexuales o bisexuales, y personas transgéneros con las mismas orientaciones. Las personas trans son homosexuales o bisexuales en un 50\%, en tanto los cisgénero lo son en un 10\% (Mazouri \& Savic, 2019).

El trabajo de Zhou et al. (1995) reveló que la stria terminalis es mayor en hombres que en mujeres, y que en mujeres transexuales es similar a mujeres cisgénero, revelando una estructura femenina en transexuales genéticamente masculinos. Savic y Arver (2011) mostró que la región parieto-temporal derecha, prefrontal inferior e ínsula poseen un mayor volumen de sustancia gris en mujeres trans en comparación con personas 
cisgénero. Una revisión de Smith et al. (2015) plantea que muchos resultados comparativos de cerebros de sujetos transexuales y cisgénero son inconsistentes y necesitan ser replicados, y los cerebros de personas trans no están completamente feminizados o masculinizados, sino más bien son estructuras o procesos cerebrales los que presentan orientaciones más femeninas o masculinas.

En otra revisión, Kreukels y Guillamon (2016) describen que el tratamiento hormonal con estrógenos en mujeres trans se asocia a una disminución del volumen cerebral acercándose a valores femeninos, que seis meses de tratamiento hormonal en mujeres trans provocó una disminución del grosor cortical y expansión de los ventrículos cerebrales y en tratamiento con testosterona para hombres trans se observó un aumento del volumen y grosor cortical, y aumento del tamaño del hipotálamo. Case et al. (2017) plantean que las personas transgéneros poseen una incongruencia entre sus rasgos físicos y la representación de su imagen corporal en la región de la corteza somatosensorial. Utilizando magnetoencefalografía registraron la actividad cerebral durante la estimulación somatosensorial del seno y la mano izquierda en personas transgéneros pre-cirugía de mujer a hombre y en mujeres cisgénero. La estimulación de la mano no mostró diferencias en la activación de la corteza somatosensorial derecha correspondiente a la mano en ambos grupos. La estimulación del seno mostró menor activación en la corteza somatosensorial correspondiente al tórax en hombres transgénero. También encontraron menor conectividad en la sustancia blanca de la ínsula anterior y el polo temporal en este grupo. Los resultados ayudan a relacionar el transgénero con diferencias en la representación neural del cuerpo y alteraciones en la conectividad de la sustancia blanca.

Un trabajo de Manzouri y Savic (2019) evaluaron el grosor de la corteza cerebral y volúmenes de estructuras subcorticales en 27 mujeres transgénero (MTrans), 40 hombres transgéneros (HTrans), 40 hombres y 40 mujeres cisgénero heterosexuales $(\mathrm{HH}$ y $\mathrm{MH}$, respectivamente) y 30 hombres y 30 mujeres cisgénero homosexuales. Los resultados muestran que la homosexualidad se relaciona con el dimorfismo sexual cerebral, en cambio la identidad de género se corresponde con redes cerebrales que procesan la percepción del propio cuerpo. Los hombres y las mujeres transgénero mostraron mayor grosor y una menor cantidad de conexiones estructurales y funcionales en la corteza cingulada-precuneus anterior y occipitoparietal dere- cha, regiones relacionadas con la percepción del propio cuerpo y del yo.

En las últimas décadas se ha avanzado mucho en el estudio de bases estructurales y funcionales cerebrales, hormonales y genéticas que podrían sustentar, en parte, la orientación sexual y la transgeneridad. Sin embargo, la formación de los futuros docentes de Educación Física, no cuentan con una perspectiva de género (Serra et al., 2020). Ayvazo y Sutherland (2009) describen la asignatura de educación física en la escuela como la que presenta más rechazo hacia la homosexualidad y transexualidad, presentando diversas agresiones verbales y comportamientos heterosexistas (Beserra et al., 2019, Piedra et al., 2016, Silva et al., 2018). También, es necesario destacar que, en la Formación Inicial Docente de los estudiantes de la carrera de Pedagogía en Educación Física se aprecia una fuerte formación biológica, pero basada principalmente en anomía, fisiología, biomecánica, entre otros (Flores y Maureira, 2019), dejando de lado temáticas como las orientaciones sexuales, identidad de género y preferencias sexuales, temas relevantes para el futuro docente y que pueden ayudar a mejorar sus propuestas didácticas, fomentando una educación más integral e inclusiva. Debido a lo mencionado anteriormente es que surgen los objetivos de la presente investigación: i) construir y validar un cuestionario para indagar sobre el conocimiento de las bases biológicas de la homosexualidad y transexualidad; ii) describir estos conocimientos en una muestra de estudiantes de Educación Física.

\section{Método}

\section{Muestra}

De tipo no probabilística intencionada. Estuvo constituida por 305 estudiantes de Pedagogía en Educación Física de tres universidades de Chile. La edad mínima fue de 18 y la máxima de 37 años, con una media de $21,7 \pm 2,9$. Del total, 114 fueron hombres $(37,4 \%)$ y 191 fueron mujeres (62,6\%). 53 estudiantes cursaban primer año de la carrera $(17,4 \%), 47$ segundo año $(15,4 \%), 88$ tercer año $(28,9 \%), 64$ cuarto año $(20,9 \%)$ y 53 quinto año $(17,4 \%)$.

\section{Instrumento}

Se elaboró un cuestionario sobre Conocimiento Biológicos de la Homosexualidad y Transexualidad (CBHT) que finalmente quedó constituido por 18 ítems (13 sobre homosexualidad y cinco sobre transexualidad) que corresponden a afirmaciones sobre aspectos cerebrales, 
hormonales y genéticos que subyacen a la homosexualidad y la transexualidad. El evaluado debe contestar $\mathrm{Co}^{-}$ rrecto, Incorrecto o No sé frente a cada ítem. Procedimiento: se realizó una búsqueda sobre los aspectos neuroanatómicos, hormonales y genéticos que podría incidir en las orientaciones sexuales e identidad de género, publicados en trabajos científicos en las últimas tres décadas. Con ello se elaboraron 24 ítems, los que fueron enviados a tres expertos que descartaron dos de ellos y realizaron correcciones en la redacción de algunos ítems. Posteriormente, el cuestionario con 22 ítems fue aplicado en forma online a una muestra piloto de 52 estudiantes de Pedagogía en Educación Física, los cuales no reportaron dudas sobre el lenguaje utilizado ni en la redacción de los ítems. Se realizaron los análisis preliminares de validez de constructo y confiabilidad del instrumento, lo que llevó a eliminar cuatro ítems los cuales correlacionaban de forma inversa con el resto del instrumento y cuya eliminación aumentaba la varianza explicada y la confiabilidad. Posteriormente se procedió a aplicar el cuestionario al total de la muestra. Los índices de validez y confiabilidad final del instrumento se presentan en el apartado de resultados. Para participar en la presente investigación todos los estudiantes firmaron un consentimiento informado.

\section{Análisis de datos}

Para determinar los índices de validez se aplicaron análisis factoriales exploratorios a través de un análisis de componentes principales con rotación ortogonal de Varimax. Para ello se utilizó el programa estadístico IBM SPSS versión 25.0 para Windows. Luego se realizaron análisis factoriales confirmatorios, donde se empleó el método de estimación de máxima verosimilitud (ML), los índices absolutos de chi cuadrado $\left(\mathrm{x}^{2}\right)$, el RMSEA (media de residuos estandarizados) y el CFI (ajuste comparado). Para ello se utilizó en programa estadístico SPSS AMOS 26.0 Los índices de confiabilidad se obtuvieron con la prueba Alfa de Cronbach. También se realizaron correlaciones de Pearson entre los diferentes factores del instrumento para valorar la relación de estos.

Para la descripción de las puntuaciones de cada ítem se utilizaron medias y desviaciones estándar. Las pruebas de normalidad de Kolmogorov-Smirnov (KS) entregaron valores $\mathrm{p}>.05$ por lo que se utilizó estadística paramétrica, con pruebas t para muestras independientes para comparar los puntajes obtenidos entre hombres y mujeres. Se consideró significativo valores $\mathrm{p}<.05$.

\section{Resultados}

\section{Propiedades psicométricas del Cuestionario CBHT}

La prueba de Kaiser-Mayer-Olkin (KMO) muestra un valor de .762 y la prueba de esfericidad de Bartlett un $p=.000$ por lo que se procedió a realizar un análisis factorial exploratorio a través de un análisis de componentes principales con rotación Varimax para identificar la estructura latente del instrumento. Este entregó la existencia de seis factores (tabla 1) que explican el $58.378 \%$ de la varianza total, con cuatro ítems que saturan en el factor 1 (genes y hormonas en la homosexualidad), cinco en el factor 2 (cerebro, homosexualidad y transexualidad), tres en el factor 3 (diferencias cerebrales homosexuales y transexuales), dos en el factor 4 (hormonas y homosexualidad), dos en el factor 5 (orientación sexual y transexualidad) y dos en el factor 6 (homosexualidad humana y animal). El alfa de Cronbach entrega un valor de .763 para los 18 ítems del instrumento.

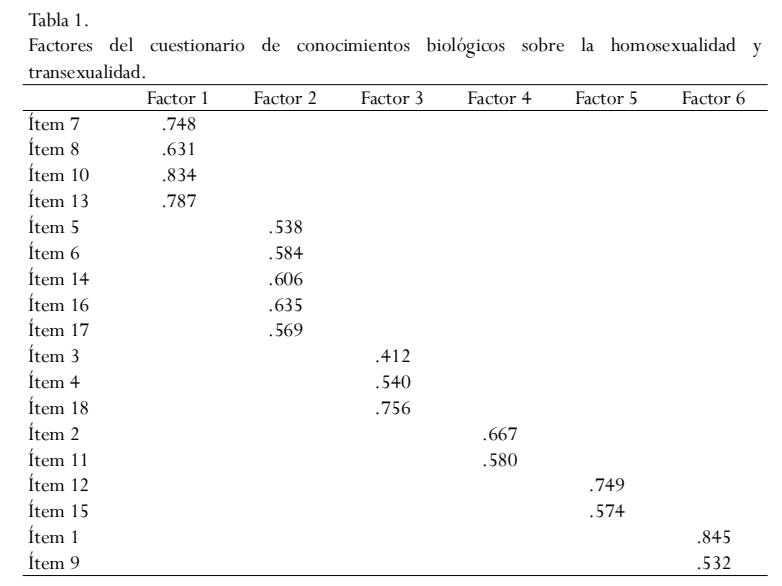

En la figura 1 se presenta el modelo final de dimensiones e ítems para el cuestionario a partir de los 6 factores extraídos del análisis factorial confirmatorio. Los resultados mostraron un $\mathrm{x}^{2}$ significativo $\left(x^{2}=256.367\right.$; $p=.000$ ), razón por la cual observamos otros valores para conocer el ajuste del modelo propuesto. Los valores del RMSEA $=.067 \mathrm{y}$ del CFI $=.828$ indican un ajuste adecuado del modelo (Maureira, 2016, Morata et al., 2015, Zubillaga \& Cañadas, 2021).

En la tabla 2 se observan las correlaciones entre las seis dimensiones del instrumento. Todas las dimensiones se asocian en forma positiva, aunque baja, donde las dimensiones 1 (genes y hormonas en la homosexualidad) y 2 (cerebro, homosexualidad y transexualidad) poseen los valores más altos de relación $(\mathrm{r}=.318 ; \mathrm{p}<.01)$, seguida por la dimensión 2 (cerebro, homosexualidad y 


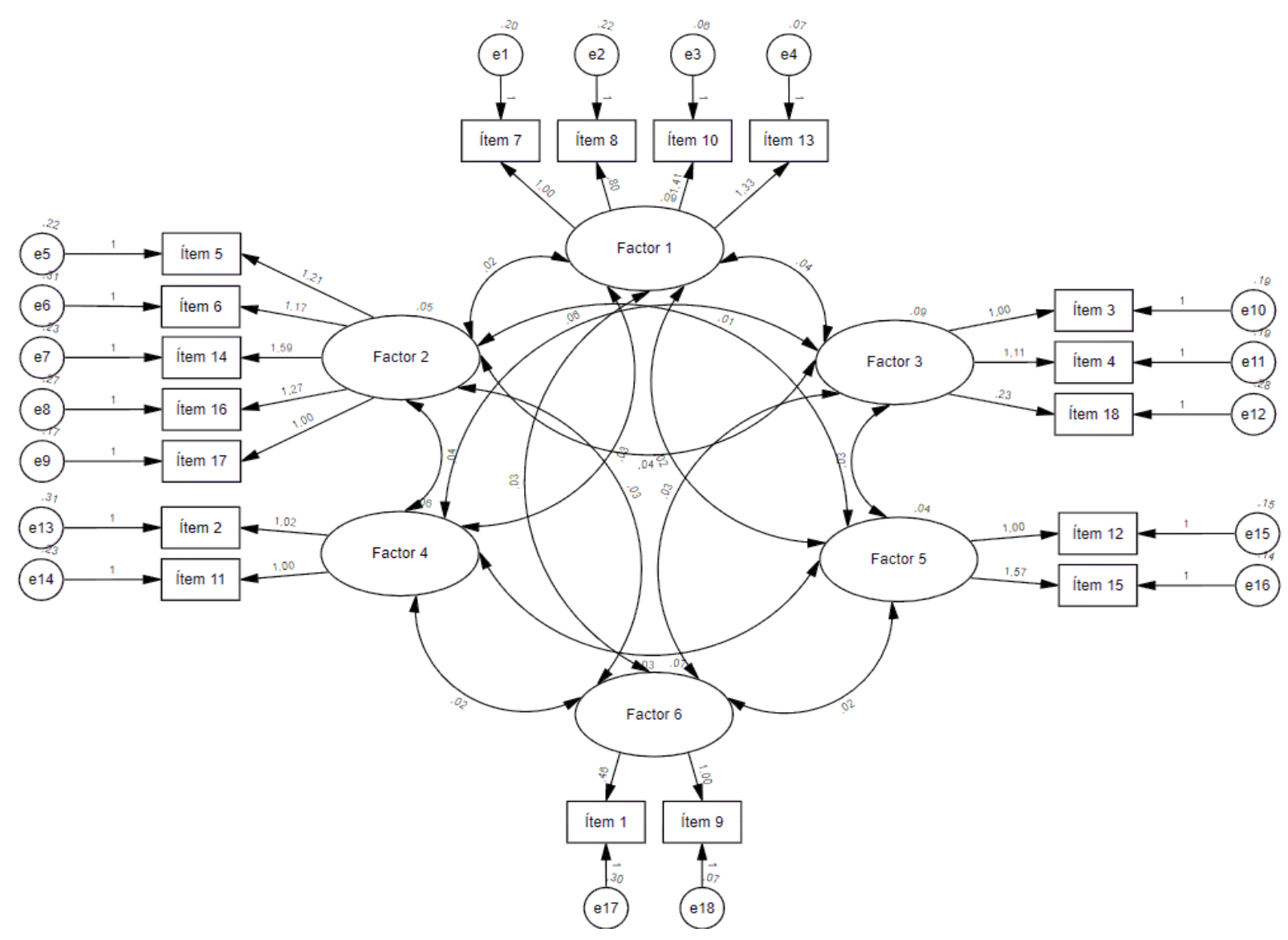

Figura 1. Análisis factorial confirmatorio.

transexualidad) y 4 (hormonas y homosexualidad) con un $\mathrm{r}=.317(\mathrm{p}<.01)$.

Tabla 2.

Correlaciones entre las dimensiones de la subescala conocimientos biológicos de la homosexualidad.

\begin{tabular}{lcccccc}
\hline & 1 & 2 & 3 & 4 & 5 & 6 \\
\hline Factor 1 & 1 & $.318 * *$ & $.274 * *$ & $.280^{* *}$ & $.188 * *$ & $.221 * *$ \\
Factor 2 & & 1 & $.255 * *$ & $.317 * *$ & $.181 * *$ & $.145 *$ \\
Factor 3 & & & 1 & $.273 * *$ & $.302 * *$ & $.203^{* *}$ \\
Factor 4 & & & & 1 & $.291 * *$ & $.144 *$ \\
Factor 5 & & & & & 1 & $.126^{*}$ \\
Factor 6 & & & & & \\
\hline$* \mathrm{p}<.05 ; * \mathrm{p}<.01$ & & & & & &
\end{tabular}

Conocimientos biológicos sobre homosexualidad y transexualidad en la muestra

En la tabla 3 se observan los porcentajes de respuestas correctas, incorrectas y no sé a cada ítem del cuestiona- rio. Es posible notar que el ítem 7. Existe un "gen gay» que determina la homosexualidad y el lesbianismo, es el que presenta mayor cantidad de aciertos con un 63.6\%, seguido del ítem 1. Ninguna otra especie animal presenta conductas homosexuales o lésbicas, con un 62.8\%. Por el contrario, el ítem 13. Cuando una mujer es lesbiana existen más probabilidad que su hermana también sea lesbiana, presenta la menor cantidad de aciertos con un .4\%, seguida de el ítem 10. Cuando un hombre es homosexual existen más probabilidad que su hermano también sea homosexual, con un 1.2\%. El ítem 9. Existen más hombres homosexuales que mujeres lesbianas, presenta la mayor cantidad de respuestas no sé, con un $84.6 \%$, seguido del ítem 12. Existen más mujeres

Tabla 3.

Porcentaje de respuestas correctas, incorrectas y no sé a cada enunciado del Cuestionario de Conocimientos Biológicos sobre Homosexualidad y Transexualidad.

Items

1. Ninguna otra especie animal presenta conductas homosexuales o lésbicas.

3. La homosexualidad y el lesbianismo presentan las mismas diferencias cerebrales.

3. La homosexualidad y el lesbianismo presentan las mismas diferencias cerebrales.
4. Los cerebros de hombres heterosexuales y homosexuales son diferentes y los cerebros de mujeres heterosexuales y lesbianas son más parecidos.

5. Existe más conocimiento cerebral sobre la homosexualidad que el lesbianismo.

6. Frente a estímulos eróticos femeninos la actividad cerebral es diferente en mujeres heterosexuales, lesbianas y bisexuales.

7. Existe un "gen gay" que determina la homosexualidad y el lesbianismo.

8. La orientación sexual estaría influenciada por las hormonas sexuales durante el embarazo.

9. Existen más hombres homosexuales que mujeres lesbianas.

10. Cuando un hombre es homosexual existen más probabilidad que su hermano también sea homosexual.

11. La predisposición genética de la homosexualidad y el lesbianismo es la misma.

12. Existen más mujeres lesbianas que mujeres bisexuales.

13. Cuando una mujer es lesbiana existen más probabilidad que su hermana también sea lesbiana.

14. La identidad de género depende de regiones cerebrales y como estas hacen que una persona perciba su cuerpo.

15. Las personas transgéneros son homosexuales o bisexuales en igual porcentaje que las personas no-transgéneros.

16. Las personas transgéneros poseen una incoherencia entre sus rasgos físicos y su imagen corporal en las regiones sensitivas del cerebro.

17. La región cerebral relacionada con el comportamiento sexual es diferente en mujeres heterosexuales y mujeres transexuales (asignados como hombres

al nacer).

18. Las regiones cerebrales relacionadas con la percepción del propio cuerpo son iguales en personas transexuales y personas no-transexuales.

\begin{tabular}{cccc} 
Respuesta & Correcto & Incorrecto & No sé \\
\hline Incorrecta & $11(3.6 \%)$ & $192(62.8 \%)$ & $102(33.6 \%)$ \\
Correcta & $47(15.4 \%)$ & $67(22.1 \%)$ & $191(62.5 \%)$ \\
Incorrecta & $18(5.9 \%)$ & $80(26.1 \%)$ & $207(68.0 \%)$ \\
Correcta & $7(2.4 \%)$ & $145(47.4 \%)$ & $153(50.2 \%)$ \\
Correcta & $29(9.5 \%)$ & $65(21.3 \%)$ & $211(69.2 \%)$ \\
Correcta & $54(17.8 \%)$ & $63(20.6 \%)$ & $188(61.7 \%)$ \\
Incorrecta & $9(2.8 \%)$ & $194(63.6 \%)$ & $102(33.6 \%)$ \\
Correcta & $5(1.6 \%)$ & $143(47.0 \%)$ & $157(51.4 \%)$ \\
Correcta & $10(3.2 \%)$ & $38(12.3 \%)$ & $258(84.6 \%)$ \\
Correcta & $4(1.2 \%)$ & $207(68.0 \%)$ & $94(30.8 \%)$ \\
Incorrecta & $22(7.1 \%)$ & $75(24.5 \%)$ & $209(68.4 \%)$ \\
Incorrecta & $16(5.1 \%)$ & $46(15.0 \%)$ & $243(79.8 \%)$ \\
Correcta & $1(.4 \%)$ & $200(65.6 \%)$ & $104(34.0 \%)$ \\
Correcta & $74(24.1 \%)$ & $40(13.0 \%)$ & $192(62.8 \%)$ \\
Incorrecta & $20(6.7 \%)$ & $56(18.2 \%)$ & $229(75.1 \%)$ \\
Correcta & $63(20.6 \%)$ & $46(15.0 \%)$ & $196(64.4 \%)$ \\
Incorrecta & $17(5.5 \%)$ & $54(17.8 \%)$ & $234(76.7 \%)$ \\
& & & \\
Incorrecta & $53(17.4 \%)$ & $36(11.9 \%)$ & $216(70.8 \%)$ \\
\hline
\end{tabular}


lesbianas que mujeres bisexuales, con un 79.8\%.

Analizando los resultados individuales de la muestra, se observa que la cantidad mínima de respuestas correctas fue de 0 y la máxima de 10 (recordemos que

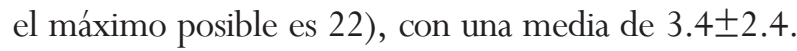
En total 29 estudiantes obtuvieron 0 respuestas correctas $(9.5 \%), 142$ obtuvieron entre 1 y 3 respuestas correctas $(46.6 \%), 98$ obtuvieron entre 4 y 6 respuestas correctas $(32.0 \%), 34$ obtuvieron entre 7 y 9 respuestas correctas (11.1\%) y 2 obtuvieron 10 respuestas correctas $(.8 \%)$.

Con relación a la cantidad de respuestas correctas, las mujeres logran una media de $3.3 \pm 2.4$ y los hombres de 3.4 \pm 2.4 , en tanto, la prueba t para muestras independientes no muestra diferencias significativas entre los grupos $(\mathrm{t}=-.340 ; \mathrm{gl}=303 ; \mathrm{p}=.734)$.

\section{Discusión}

El cuestionario de conocimientos biológicos de la homosexualidad y transexualidad ha mostrado ser un instrumento válido y confiable para recoger información sobre aspectos genéticos, hormonales y cerebrales que podrían encontrarse a la base de las orientaciones sexuales e identidad de género. El instrumento presenta una estructura de seis factores con una adecuada varianza explicada y una buena consistencia interna, por lo cual este cuestionario constituye una importante, y quizás la primera herramienta, para evaluar este tipo de conocimiento en estudiantes de Pedagogía. Las orientaciones sexuales e identidad de género son ámbitos fundamentales donde el docente debe conocer no sólo aspectos socio-culturales, sino también biológicos, que lo ayuden a concientizarse sobre estos temas, ya que dicho conocimiento reduce los niveles de heterosexismo y la homofobia en sus clases (García et al., 2013).

En relación con los resultados que entregó el instrumento en estudiantes de Educación Física, el hecho de que no se haya identificado un gen gay o que existen otras especies animales homosexuales o bisexuales son los únicos conocimientos que contestaron correctamente más del 50\% de la muestra. El hecho que la predisposición genética sea diferente para la homosexualidad y el lesbianismo o que la identidad de género dependa de ciertas regiones del cerebro lograron casi el 25\% de respuestas correctas. En 14 de los 18 ítems más del $50 \%$ de los estudiantes contestan no saber, lo que da cuenta de una amplia falta de conocimientos sobre estos temas, además de que el $78.6 \%$ de la muestra obtuvo sólo entre una y seis respuestas correctas (de un máxi- mo de 18). Esto revela la necesidad de incorporar contenidos biológicos sobre orientación sexual e identidad de género durante la formación de futuros docentes, ya que como revela un estudio de Flores y Maureira (2020) sólo 9 de 26 universidades que dictan la carrera de Pedagogía en Educación Física en Chile poseen asignaturas sobre neurociencia y sólo tres de ellas poseen un enfoque educativo. Sin embargo, ninguna de ellas incluye contenidos sobre orientación sexual e identidad de género. Un estudio de (1992) investigó el efecto de información sobre el desarrollo de la orientación sexual sobre las actitudes hacia la homosexualidad, revelando que el grupo experimental presento puntajes más bajos de homofobia que el grupo control, con una mayor disminución en mujeres. Recordemos que para Ayvazo y Sutherland (2009) la Educación Física es el área que presenta más rechazo a la diversidad sexual y que para Fernández (2009) un mayor conocimiento sobre las bases biológicas de la homosexualidad y transexualidad disminuyen las actitudes homofóbicas y transfóbicas.

\section{Conclusión}

El cuestionario de conocimientos biológicos de la homosexualidad y transexualidad posee índices adecuados de validez y confiabilidad en una muestra de estudiantes de Educación Física. Su aplicación revela gran desconocimiento de estos temas en estos futuros docentes, ya que más de 50\% contestó no sé en 14 de los 18 ítems del cuestionario y el 78.6\% de la muestra obtuvo sólo entre una y seis respuestas correctas de un máximo de 18 . No se observaron diferencias en la cantidad de respuestas correctas entre hombres y mujeres.

Son necesarias investigaciones en muestras de mayor tamaño y de diferentes universidades, para tener una mirada más amplia de estos conocimientos biológicos en estudiantes de Educación Física en todo Chile. Además, este cuestionario se podría utilizar para recoger información en otras carreras de Pedagogía y en profesores que actualmente ejercen en el contexto escolar, de manera de profundizar en las necesidades de formación y actualización de estos profesionales, permitiendo una mayor normalización de las orientaciones sexuales e identidad de género en las aulas.

\section{Referencias}

American Psychological Association (2011). Definition of terms: Sex, Gender, Gender Identity, Sexual Orientation. Disponible en: http://www.apa.org/pi/lgbt/ 
resources/sexualitydefinitions.pdf

Annicchiarico, I. (2009). Psicobiología de la homosexualidad masculina: hallazgos recientes. Univ $P_{\text {sychol, }}$ 8(2), 429-446.

Ayvazo, S. \& Sutherland, S. (2009). Uncovering the secrets: Homophobia in Physical Education. Action in Teacher Education, 31(3), 56-69. https: //doi.org/ 10.1080/01626620.2009.10463528

Bagemihl, B. (1999). Biological exuberance: animal homosexuality and natural diversity. St. Martin's Press.

Bailey, J. \& Pillard, R. (1991). A genetic study of male sexual orientation. Arch Gen Psychiatry, 48(12), 10891096. https://doi.org/10.1001/ archpsyc. 1991.01810360053008

Balthazart J. (2020). Sexual partner preference in animals and humans. Neurosci Biobehav Rev, 115, 34-47. https:/ /doi.org/10.1016/j.neubiorev.2020.03.024

Beserra, J., Brito, A. \& Ribeiro, S. (2019). Homofobia nas aulas de educação física: um desafio para os professores de educação física do município de Buriti dos montes - Piaui. Revista do Plano Nacional de Formação de Professores da Educação Básica, 7(2), 81-90.

Case, L., Brang, D., Landazuri, R., Viswanathan, P. \& Ramachandran, V. (2017). Altered white matter and sensory response to bodily sensation in female-tomale transgender individuals. Arch Sex Behav, 46(5), 1223-1237. https://doi.org/10.1007/s10508-0160850-Z

Clay, Z. \& deWaal, F. (2015). Sex and strife: post-conflict sexual contacts in bonobos. Behaviour, 152(3-4), 313334. https://doi.org/10.1163/1568539X-00003155

Clay, Z. \& Zuberbühler, K. (2011). Bonobos extract meaning from call sequences. PLoS ONE, 6(4), e18786. https://doi.org/10.1371/ journal.pone.0018786

Coria-Ávila, G. (2012). The role of conditioning on heterosexual and homosexual partner preference in rats. Socioaffective Neuroscience \& Psychology, 2(1), 17340. https://doi.org/10.3402/snp.v2i0.17340

Fernández,A. (2009). Bases biológicas de la preferencia sexual. Ciencia, 60(2), 23-36.

Flores, E. \& Maureira, F. (2020). Formación pedagógica en las carreras de educación física: falta de conocimientos para un profesional del siglo XXI. EmásF, Revista Digital de Educación Física, 11(62), 118-126.

Ganna, A., Verweij, K., Nivard, M., Maier, R. \&Wedow, R. (2019). Large-scale GWAS reveals insights into the genetic architecture of same-sex sexual behavior. Science, 365, eaat7693. https://doi.org/10.1126/ science.aat 7693
García, P. (2005). Identidad de género: modelos explicativos. Escritos de Psicología, 7, 71-81.

García, R., Sala, A., Rodríguez, E. \& Sabuco, A. (2013). Formación inicial del profesorado sobre género y coeducación: impactos metacognitivos de la inclusión curricular transversal sobre sexismo y homofobia. Revista de Curriculum y Formación del Profesorado, 17(1), 269-287.

Hines, M. (2011). Gender development and the human brain. Annu Rev Neurosci, 34, 69-88. https: / / doi.org/ 10.1146/annurev-neuro-061010-113654

Kinnunen, L., Moltz, H., Metz, J. \& Cooper, M. (2004). Differential brain activation in exclusively homosexual and heterosexual men produced by the selective serotonin reuptake inhibitor, fluoxetine. Brain Research, 1024, 251-254. https://doi.org/ 10.1016/j.brainres.2004.07.070

Kreukels, B. \& Guillamon, A. (2016). Neuroimaging studies in people with gender incongruence. Int Rev Psychiatry, 28(1), 120-128. https://doi.org/10.3109/ 09540261.2015 .1113163

Landarroitajauregi, J. (2010). Reflexiones críticas para sexólogos avezados. Revista Española de Sexología, 1, 157-158.

Langström, N., Rahman, Q., Carlström, E. \& Lichtenstein, P. (2010). Genetic and environmental effects on same-sex sexual behavior: a population study of twins in Sweden. Archives of Sexual Behavior, 39(1), 75-80. https://doi.org/10.1007/s10508-0089386-1

LeVay, S. (1991). A difference in hypothalamic structure between heterosexual and homosexual men. Science, 253, 1034-1037. https://doi.org/10.1126/ science. 1887219

Manzouri, A. \& Savic, I. (2018). Cerebral sex dimorphism and sexual orientation. Hum Brain Mapp, 39(3), 1175-1186. https://doi.org/10.1002/ hbm. 23908

Manzouri, A. \& Savic, I. (2019). Possible neurobiological underpinnings of homosexuality and gender dysphoria. Cereb Cortex, 29(5), 2084-2101. https:// doi.org/10.1093/cercor/bhy090

Maureira, F. (2016). Estadística avanzada para educación física. Editorial Académica Española.

Maureira, F. (2020). Amor, sexo y cerebro. Bubok Publishing. Morata, M., Holgado, F., Barbero, I. \& Méndez, G. (2015). Análisis factorial confirmatorio. recomendaciones sobre mínimos cuadrados no ponderados en función del error tipo I de ji-cuadrado y RMSEA. Acción Psicológica, 12(1), 79-90. https://dx.doi.org/ 
10.5944/ap.12.1.14362

Morris, J., Jordan, C. \& Breedlove, S. (2004). Sexual differentiation of the vertebrate nervous system. Nat Neurosci, 7, 1034-1039. https://doi.org/ $10.1038 / \mathrm{nn} 1325$

Piedra, J., Ramírez, G., Ries,F., Rodríguez,A. \& Phipps C. (2016). Homophobia and heterosexism: Spanish physical education teachers' perceptions. Sport in Society, 19(8-9), 1156-1170. https://doi.org/ 10.1080/17430437.2015.1096257

Piskur, J. \& Degelman, D. (1992). Effect of reading a summary of research about biological basis of homosexual orientation on attitudes toward homosexuals. Psychological Reports, 71, 1219-1225. https://doi.org/10.2466/pr0.1992.71.3f.1219

Platero, R. (2014). Transexualidades. Bellaterra.

Rosalba, A. (2016). Androginia: la identidad de género no binaria en el individuo. Revista Anual del CINAVESAY, 6, 65-71.

Savic, I. \& Arver, S. (2011). Sex dimorphism of the brain in male-to-female transsexuals. Cerebral Cortex, 21, 2525-2533. https://doi.org/10.1093/cercor/ bhr032

Savic, I., Berglund, H. \& Lindstrom, P. (2005). Brain response to putative pheromones in homosexual men. PNAS, 102(20), 7356-7361. https://doi.org/ 10.1073/pnas.0407998102

Savic, I., García-Falgueras, A. \& Swaab, D. (2010) Sexual differentiation of the human brain in relation to gender identity and sexual orientation. Prog Brain Res, 186, 41-62. https://doi.org/10.1016/B978-0444-53630-3.00004-X

Serra, P., Prat, M., Nicolino, A., Soler, S. \& Silva, A. (2020). Género y curriculum de formación del profesorado en Educación Física: un diálogo entre Brasil y España. Revista Iberoamericana de Educación, 82(2), 191-210. https: / / doi.org/10.35362/rie8223637
Silva, P., Jaeger, A. \& Valdivia, P. (2018). Percepción de los estudiantes sobre comportamientos homofóbicos y heterosexistas en educación física. Revista de Psicología del Deporte, 27(2), 39-46.

Smith, E., Junger, J., Derntl, B. \& U. Habel, U. (2015). The transsexual brain - A review of findings on the neural basis of transsexualism. Neuroscience and Biobehavioral Reviews, 59, 251-266. https://doi.org/ 10.1016/j.neubiorev. 2015.09.008

Soley, P. (2014). Transexualidad y Transgénero: una perspectiva bioética. Revista de Bioética y Derecho, 30, 2139. https://doi.org/10.1344/rbd2014.30.9904

Swaab, D. \& Hofman, M. (1990). An enlarged suprachiasmatic nucleus in homosexual men. Brain Research, 537(1-2), 141-148. https://doi.org/ 10.1016/0006-8993(90)90350-k

Vasey, P., Foroud, A., Duckworth, N. \& Kovacovsky, S. (2006). Male-female and female-female mounting in Japanese macaques: A comparative analysis of posture and movement. Archives of Sexual Behavior, 35, 116-128. https://doi.org/10.1007/s10508-0059007-1

Wang, Y., Wu, H. \& Sun, Z. (2019). The biological basis of sexual orientation: How hormonal, genetic, and environmental factors influence to whom we are sexually attracted. Front Neuroendocrinol, 55, 100798. https://doi.org/10.1016/j.yfrne.2019.100798

Zhou, J., Hofman, M., Gooren, L. \& Swaab, D. (1995). A sex difference in the human brain and its relation to transsexuality. Nature, 378(6552), 68-70. https: // doi.org/10.1038/378068a0

Zubillaga, M. \& Cañadas, L. (2021). Diseño y validación del cuestionario «\#EvalEF» para conocer el proceso de evaluación desarrollado por los docentes de educación física. Retos, 42, 47-55. https://doi.org/ $10.47197 /$ retos.v42i0.86627

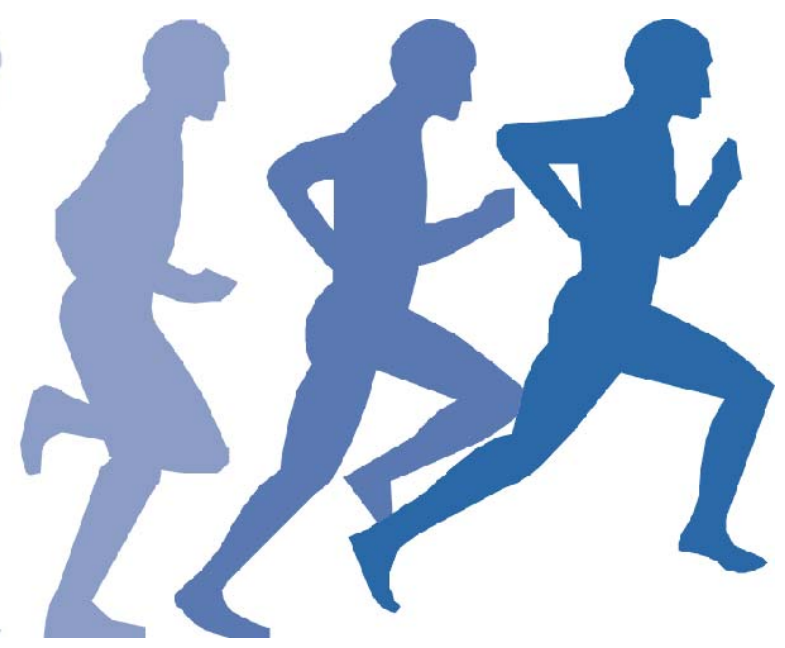

